\title{
Microwave Dielectric Property Based Classification of Malignancies
}

\author{
Tuba Yilmaz \\ Department of Electronics and Communication Engineering, \\ Istanbul Technical University, \\ Istanbul, Turkey \\ tuba.yilmaz@itu.edu.tr
}

\begin{abstract}
Broadband dielectric property measurements of biological tissues is mostly performed with open ended coaxial probe technique due to a number of advantages such as flexible sample shape and size. However, the technique is known to suffer from high error rates; thus, envisioned applications of the technique remains hampered by this problem. One way to mitigate such error for medical applications is to perform tissue classification with machine learning algorithms. In this work, Cole-Cole parameters of rat liver dielectric properties are used for training and testing of an in house Support Vector Machine (SVM) algorithm to enable malignant tissue classification. ColeCole parameters are fitted with Particle Swarm Optimization (PSO) to a total of 700 dielectric property measurements collected from 22 rats. The Cole-Cole parameters are fed to the SVM algorithm and k-fold cross validation is used to prevent the algorithm from memorizing the data. Hepatic malignancies are classified with $96 \%$ accuracy where a better accuracy is obtained in comparison to plain dielectric property measurement and also an automated decision making mechanism is enabled.

Index Terms - component, formatting, style, styling, insert
\end{abstract}

\section{INTRODUCTION}

The need to characterize the dielectric properties of healthy and malignant tissues is emerged due to the potential diagnosis and treatment applications of microwaves such as hyperthermia and ablation [1]. Therefore, the malignant and healthy dielectric properties of animal and human liver tissues is widely studied in the literature with open ended coaxial probes to mostly exploit the potential microwave applications [2], [3]. The open ended coaxial probe technique mostly utilized to measure the dielectric properties of high permittivity and high loss materials due to the flexible sample size requirements, convenient measurement procedure, nondestructive and broadband measurement capabilities. Although the open ended coaxial probe technique is successfully utilized in laboratory environment, the technique suffers from low measurement accuracy and low measurement repeatability rates. For commercial probes the measurement error can be as high as 5\% [4]. While many different practical applications are envisioned for the technique in the literature, these shortcomings of the open ended coaxial measurement method limits the technique's utilization with laboratory use [5], [6]. The contributing error sources include the inversion algorithms used for dielectric

This project has received funding from the MyWave COST Action COST CA17115 and Istanbul Technical University Grant 41554. property retrieval and errors stemming from the experimental set-up. In the laboratory environment the probe is usually fixed in a position and the material under test is placed at the tip of the probe. However, for a practical application the probe can not be fixed rather it should be freely moved. Therefore, in a practical setting the error due to the experimental setting is expected to increase.

To realize a practical application, such as a microwave biopsy device, the technique should be able to differentiate between benign and malignant or healthy and malignant tissue with high precision. Thus, instead of decreasing the error associated with dielectric property measurement one can focus on accurate differentiation of the malignant tissues from others. In this paper, SVM algorithm is adopted to classify the malignant and other tissues to enable possible practical applications of open ended coaxial probe technique. Dielectric property measurement data collected from rat liver tissues between 500 $\mathrm{MHz}$ to $6 \mathrm{GHz}$ are categorized as malignant, cirrhotic, benign, and healthy. For each measurement, single pole Cole-Cole parameters are calculated. Calculated parameters are then fed to the classification algorithm.

\section{Materials AND Methods}

\section{A. Dielectric Property Measurements}

Before dielectric property measurement, the probe is calibrated with open, short, and known material protocol. Through a midline incision the animal's liver is accessed, while keeping the hepatic vein contact the dielectric property measurements were performed. Before a dielectric property measurement is taken the probe tip is calibrated, cleaned and air dried. A dielectric property measurement is taken first then the measurement site is wiped with $0.9 \%$ saline solution, these measurements are named wet and dry, respectively.

\section{B. Classification Algorithm}

The measurements recorded and categorized based on the pathology results. Over 700 measurements were collected. The measurements are performed between $500 \mathrm{MHz}$ to $6 \mathrm{GHz}$ with 12 sweep points. With particle swarm optimization (PSO) the Cole-Cole parameters are calculated. The 12 data points is reduced to 6 variables due to Cole-Cole parameters. Note that in a laboratory setting the sweep points are usually in the 
order of $100-150$. Thus, when the other dielectric property measurements are considered the data points would be reduced greatly. Then the data is randomly distributed into 10 groups. One group is used for testing the algorithm and the rest is used for training.

\section{RESULTS}

\section{A. Measurement Results}

In Fig. 1 median dielectric property measurements of malignant and healthy liver tissues are given. The inherent relative permittivity discrepancy between the malignant and healthy tissues can also be observed from the graph.It is worth noting that although the median of the measurements are differentiable, part of the measurements are located at rather low or high end of the measured values and thus they could be confused for being malign or benign.

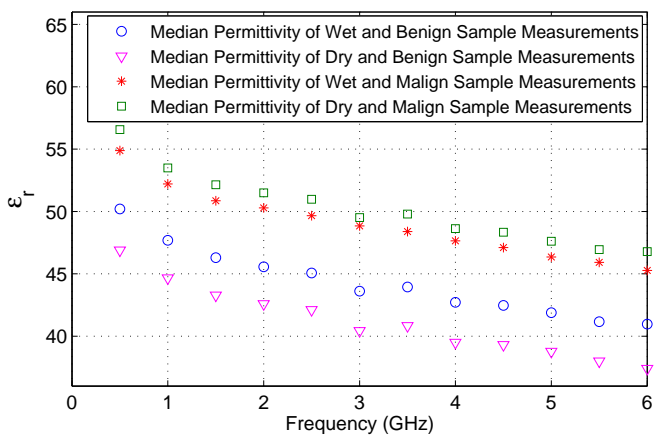

(a)

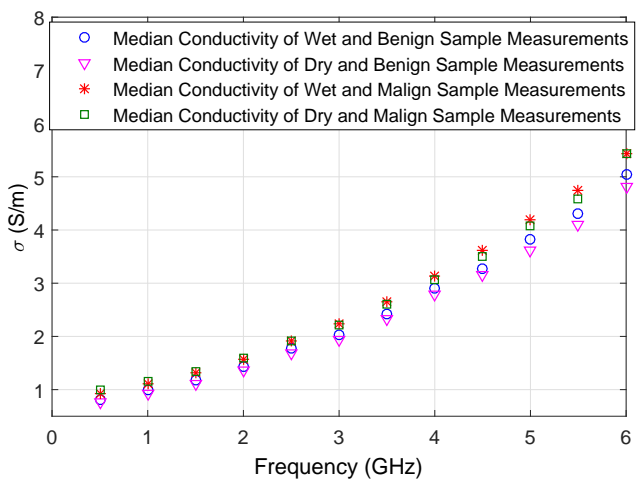

(b)

Fig. 1. Dielectric property measurement results (a) averaged measured relative permittivity of benign and malignant rat liver tissue (b) averaged measured conductivity of benign and malignant rat liver tissue.

\section{B. Comparison with the ROC curves}

The ROC curves are calculated by averaging the measured permittivity at the given frequency range. First the thresholds for wet and dry measurement samples are designated by using the fitted $\delta \epsilon$ for each group. For wet samples, four thresholds were designated between 40.65 and 49.42 by equally spacing the parameters. Then classification is performed by comparing the averaged permittivity for each measurement to the designated threshold. Then sensitivity and specificity are calculated. Same procedure is repeated for dry measurements where the thresholds are chosen based on the fitted Delta epsilon parameters to Dry measurements; that is, 39.4 and 47.93.

As it can be seen from the figure sensitivity and specificity are dependent on the selection of the threshold. Moreover, as the sensitivity is increased the specificity decreases and vice versa. For wet samples maximum sensitivity is $96 \%$ where at the same threshold the specificity is $13 \%$, the maximum specificity is $82 \%$ and for the same threshold the sensitivity is $42 \%$. For Dry samples maximum sensitivity is $93 \%$ and for the same threshold the specificity is $37 \%$, similarly maximum specificity is $77 \%$ and for the same threshold the sensitivity is $91 \%$.

\section{CONCLUSION}

The malignant and benign as well as malignant and healthy tissues can be differentiated based on their dielectric property discrepancies. However, accurate measurement of broadband dielectric properties is difficult to achieve due to the inherent error of open ended coaxial probe technique. However, this error can be mitigated by adopting the classification algorithms; that is, training the algorithm and then estimating which class the incoming data belong to. In this paper, rat liver dielectric properties are measured between $500 \mathrm{MHz}$ to $6 \mathrm{GHz}$ with 12 data points. Then, Cole-Cole parameters are fitted to the dielectric measurement data. By feeding the Cole-Cole data to the classification algorithm, the algorithm is trained and tested. The classification algorithm was able to estimate the class of the incoming data with $96 \%$ accuracy.

\section{REFERENCES}

[1] A. P. O'Rourke, D. Haemmerich, P. Prakash, M. C. Converse, D. M. Mahvi, and J. G. Webster, "Current status of liver tumor ablation devices," Expert Review of Medical Devices, vol. 4, no. 4, pp. 523-537, 2007. [Online]. Available: http://dx.doi.org/10.1586/17434440.4.4.523

[2] D. Haemmerich, S. T. Staelin, J. Z. Tsai, S. Tungjitkusolmun, D. M. Mahvi, and J. G. Webster, "In vivo electrical conductivity of hepatic tumours," Physiological Measurement, vol. 24, no. 2, p. 251, 2003. [Online]. Available: http://stacks.iop.org/0967-3334/24/i=2/a=302

[3] A. P. O'Rourke, M. Lazebnik, J. M. Bertram, M. C. Converse, S. C. Hagness, J. G. Webster, and D. M. Mahvi, "Dielectric properties of human normal, malignant and cirrhotic liver tissue: in vivo and ex vivo measurements from 0.5 to $20 \mathrm{ghz}$ using a precision open-ended coaxial probe," Physics in Medicine and Biology, vol. 52, no. 15, p. 4707, 2007. [Online]. Available: http://stacks.iop.org/0031-9155/52/i=15/a=022

[4] Keysight. (2017) Keysight 85070E Dielectric Probe Kit 200 $\mathrm{MHz}$ to $50 \mathrm{GHz}$ technical overview. [Online]. Available: http://literature.cdn.keysight.com/litweb/pdf/5989-0222EN.pdf

[5] T. Yilmaz, M. A. Kılıç, M. Erdoğan, M. Çayören, D. Tunaoğlu, İsmail Kurtoğlu, Y. Yaslan, H. Çayören, A. E. Arıkan, S. Teksöz, G. Cancan, N. Kepil, S. Erdamar, M. Özcan, İbrahim Akduman, and T. Kalkan, "Machine learning aided diagnosis of hepatic malignancies through in vivo dielectric measurements with microwaves," Physics in Medicine and Biology, vol. 61, no. 13, p. 5089, 2016. [Online]. Available: http://stacks.iop.org/0031-9155/61/i=13/a=5089

[6] W. Anderson, "Microwave biopsy probe," Patent, 13, 2006, uS20060079774 A1. [Online]. Available: https://www.google.com/patents/US20060079774 